\title{
Biodiesel Production from Spent Coffee Grounds Oil
}

\author{
Chelselyn Chuaca, Elza Karenina, Kezia Yusuf, Shafwah Dzahabiyya, \\ Alwan Raihan, Evita Legowo, Hery Sutanto* \\ Chemical Engineering Department, Faculty of Life Sciences and Technology, Swiss German University, Kota \\ Tangerang, 15143, Indonesia \\ ${ }^{*}$ Corresponding author. Email: hery.sutanto@sgu.ac.id
}

\begin{abstract}
Biodiesel has been considered as an alternative fuel to replace conventional oil in the world. Biodiesel development has reached blending for $30 \%$ and has been implemented by Indonesia since 2020. Sources for biodiesel production vary, as is well known, mostly from CPO, raw vegetable oil, used cooking oil, and animal fats. The utilization of waste as raw material for biodiesel can provide additional energy while reducing the amount of existing waste. Spent coffee grounds is an alternative raw material for biodiesel production. The coffee consumption in Indonesia in 2020 increased to $13.9 \%$ compared to 2019 which leads to a large amount of unutilized spent coffee grounds. This research focused on producing biodiesel from Indonesian commercial RTD coffee and Indonesian freshly brewed coffee; the coffee oil was extracted from spent coffee grounds which later the coffee oil became the main component for the biodiesel production process. The direct esterification showed a promising result for biodiesel production with Fatty Acid Methyl Ester (FAME) content $78.7 \%$ for commercial RTD coffee and $69.2 \%$ for freshly brewed coffee sample by varying methodologies for both sample direct esterification and direct transesterification.
\end{abstract}

Keywords: Biodiesel, Esterification, Fatty acid methyl ester, Spent coffee grounds, Transesterification

\section{INTRODUCTION}

Fossil fuel is used a lot for commercial purposes, mainly for electricity, transportation fuel, and heating. However, the consumption of fossil fuel has been an issue for these past years as it gives more potential for global warming and limited availability (US EPA, 2021). The disadvantages of fossil fuels lead researchers to focus on replacing fossil fuels with alternative energy to become sustainable, renewable, and produce clean energy, for instance biodiesel.

The development of biodiesel in Indonesia continues to grow in the past decade to achieve more efficient and cleaner biodiesel. Researchers focused to optimize the production of biodiesel and also to utilize a new source of biodiesel derived waste. One of the examples is production of biodiesel from waste cooking oil. Optimizing waste as a source of biodiesel has the goal to reduce the cost of raw material for biodiesel production.

Spent coffee grounds (SCG) or coffee waste has the potential to be one of the alternative sources of biodiesel production. Indonesia is number 4th in the world with the most production of coffee in the world. Coffee consumption has increased continuously worldwide resulting in abundant raw material of spent coffee grounds. In Indonesia coffee consumption is increasing $10 \%$ from 2013 - 2017 and predicted to increase in upcoming years [1]. However, the utilization of the spent coffee grounds is very low while spent coffee grounds are abundant. According to previous research the yield conversion of spent coffee grounds into biodiesel is $13 \%$ [2].

This research will provide an alternative new raw material for biodiesel production from waste. Due to the increase of coffee consumption and production worldwide, thus coffee has the potential to become a source of biodiesel. This research is limited to SCG feedstock obtained from a single freshly brewed and one brand for commercial RTD coffee. The aim of this research is to produce biodiesel from spent coffee grounds and to discover suitable methodology for Indonesian spent coffee ground to produce biodiesel 


\section{MATERIALS AND METHOD}

\subsection{Materials and Equipment}

The materials used for this experiment will be nhexane, methanol, potassium hydroxide as an alkali catalyst. The extraction process is using reflux extraction. $\mathrm{N}$ hexane will be used as the solvent for the extraction of coffee oil. The chemicals required to conduct this experiment is post analytic grade for further analytical purposes. The n-hexane (Merck, Germany for analysis catalogue number 1.04367.2500). The methanol (Merck, Germany catalogue number 1.06009.5200) used for transesterification reaction. The last chemical materials used is potassium hydroxide (Merck, Germany catalogue 1.05033 .1000 with minimum purity $85 \%$. Biodiesel as a blank sample in GCMS analysis obtained from LEMIGAS from December 2018).

Soxhlet apparatus used for the extraction of spent coffee grounds to produce coffee oil. In order to recover solvents after the extraction process, a vacuum evaporator was conducted in Swiss German University laboratory with the evaporator performed under moderate vacuum conditions. Centrifuge has been used during the separation of transesterification reactions. The gas chromatography-mass spectrometry (GCMS) used Agilent Technologies $6890 \mathrm{~N}$ running with inlet temperature $250^{\circ} \mathrm{C}$ with helium used as mobile phase

\subsection{Methods}

The design of this experiment will be explained in the flow diagram chart in Figure 1. The spent coffee grounds were prepared 1 week before the drying process for both samples to ensure freshness of the sample. After both samples dried overnight at $105^{\circ} \mathrm{C}$ to reduce the moisture content, the samples were prepared for the extraction process. The first step of extraction was through the reflux process. The extracted coffee oil containing hexane was separated with a rotary evaporator. As a result, hexane was recovered for the next batch of production. The coffee oil was analysed by GCMS to observe the fatty acid compositions. During the GCMS analysis both sachet and freshly brewed coffee samples are conducted in the biodiesel production process and later be observed again by GCMS whether the fatty acid methyl ester was formed.

\subsubsection{Spent Coffee Grounds Collection}

The spent coffee grounds from freshly brewed were collected from 'KRAH Coffee and Cuisine', South Tangerang. The SCG is derived from a Gayo blend mixture of Arabica coffee with different variations that has been used in the freshly brewed for making espressobased beverages. Pressure around 9 bar and temperature $90-95^{\circ} \mathrm{C}$ are required for the coffee machine to produce espresso from KRAH Coffee.

The conventional coffee used in this research was obtained from a commercial coffee that contains spent coffee grounds produced. The coffee can be found easily in supermarkets or small grocery stores all over Indonesia. The brewing method of commercial RTD coffee only requires hot water. The amount of water used was following the instruction given, which is $25 \mathrm{~g}$ commercial RTD coffee poured with $250 \mathrm{ml}$ boiled water.

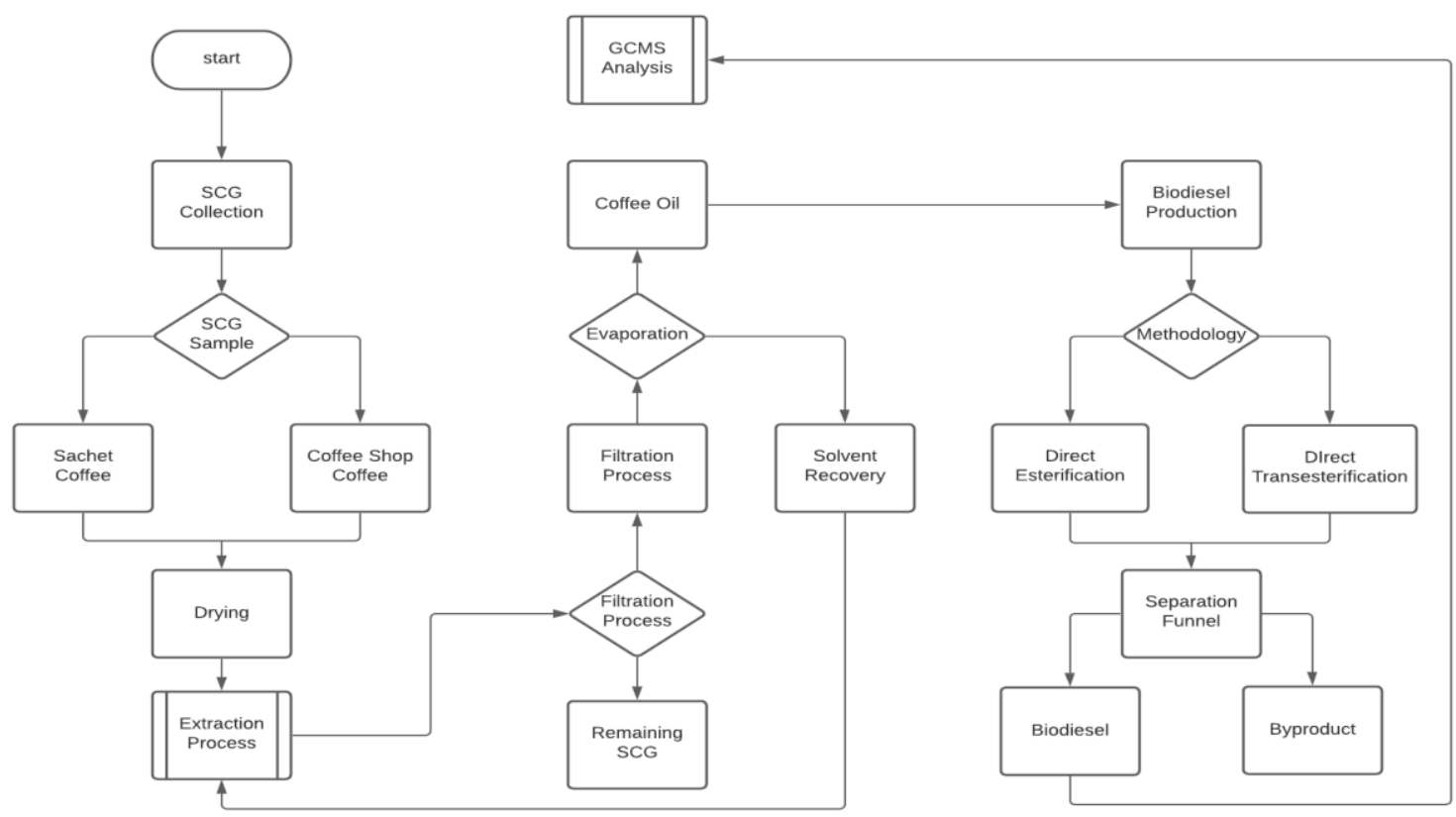

Figure 1. Experiment mechanism diagram 
The selected commercial RTD coffee that contains spent coffee grounds with the consideration becomes the sample of majority Indonesian commercial RTD coffee consumption. Since the consumption of freshly brewed mainly appears in bigger cities, such as Jakarta and Bandung. Meanwhile, the commercial RTD coffee consumption is higher compared to ground coffee due to cheaper cost (respectively), shorter preparation time, and easier to make [3]. The consumers of commercial RTD coffee are mainly households. The commercial RTD coffee used in this research is a brand product with $100 \%$ pure coffee. Meanwhile, the other brand mainly sold mixed coffee with sugar. The sugar composition in commercial RTD coffee is considered to affect the fatty acid produced which is the aim for this research.

\subsubsection{Oil Extraction Process}

The coffee oil was extracted with method based on literature using Soxhlet apparatus as the extraction container [4]. N-hexane was utilized as a solvent with the SCG and solvent ratio 1:3. The solvent used was post analytic grade for further characteristic and analysis. The extracted oil was separated using a rotary evaporator at $55^{\circ} \mathrm{C}$ with $50 \mathrm{rpm}$ under partial vacuum condition $(0.5$ bar). The separated solvent was recovered and could be used for the next batch of production. The coffee oil produced was divided for biodiesel production methodologies. The coffee oil yield can be calculated by using Eq. 1 and the hexane recovery yield by Eq.2.

Coffee Oil Yield $=\frac{\text { ml coffee oil }}{\text { gram coffee }} \times 100 \%$ (1)

Hexane Recovery yield $=$

$\frac{\text { hexane volume in coffee oil }}{\text { total hexane used }} \times 100 \%$

\subsubsection{Transesterification Reaction}

Coffee oil samples from freshly brewed and commercial RTD coffee were used for the transesterification reaction. The coffee oil was reacted with potassium hydroxide and methanol in the transesterification process. The optimization of transesterification reaction will be achieved $15 \%$ triglycerides from the coffee powder with parameters of $1.5 \%$ wt $\mathrm{KOH}$ and $40 \%$ vol methanol with the reflux time of 15 minutes at $75^{\circ} \mathrm{C}$ [4].

\subsubsection{Esterification Reaction}

The pure coffee oil was used for the direct esterification reaction. The reaction of esterification was reacted by fatty acid with alcohol to produce FAME as explained in Figure 1. Both commercial RTD coffee and freshly brewed coffee were esterified and both products were separated from the oil formation and the by-product utilizing separation funnel. After pure oil was separated, the oil was analysed through GCMS analysis to check the trace of FAME formation in the samples. The esterification reaction was conducted with the reaction parameters of 1:9 $\left(1 \mathrm{~g} \mathrm{SCG}\right.$ : $\left.9 \mathrm{ml} \mathrm{MeOH}-\mathrm{H}_{2} \mathrm{SO}_{4}\right)$ using a reflux method at $65^{\circ} \mathrm{C}$ for 3 hours [4].

\subsubsection{Biodiesel Oil Analysis}

The biodiesel produced in this research is analysed using GCMS analysis performed in PUSLABFOR (Indonesian Police Forensic Laboratory) located in Kalimalang, East Jakarta. The aim of GCMS analysis is to collect information of fatty acids or fatty acid methyl compositions ester as the component of biodiesel.

\section{RESULTS AND DISCUSSION}

\subsection{Coffee Oil Extraction}

Subsequently, the product containing coffee oil and solvent were separated using a rotary evaporator, the separation method is based on boiling point difference (Table 1). Under vacuum conditions, the boiling point of hexane is $53^{\circ} \mathrm{C}$ [5]. The conditions of the evaporation process were performed under partial vacuum $(0.5 \mathrm{bar})$ at $50 \mathrm{rpm}$ with cooling water inlet at $23.5^{\circ} \mathrm{C}$. The evaporation kept proceeding until the hexane was completely evaporated.

Table 1. Evaporation results of the coffee samples

\begin{tabular}{ccc}
\hline & $\begin{array}{c}\text { Commercial } \\
\text { RTD coffee }\end{array}$ & $\begin{array}{c}\text { Freshly } \\
\text { brewed coffee }\end{array}$ \\
\hline Oil yield $(\%)$ & $8.5 \pm 0.5$ & $11.0 \pm 1.0$ \\
\hline $\begin{array}{c}\text { Solvent recovery } \\
\text { yield }(\%)\end{array}$ & 23 & 33 \\
\hline Color of oil & dark brown & light brown \\
\hline
\end{tabular}

In this experiment around 15-30 minutes was required to complete the evaporation process, which applies for both samples (commercial RTD coffee and freshly brewed coffee). After the separation has been completed, the $n$-hexane was recovered and could be used for the next batch of extraction process. On the other side, the coffee oil would be used as the main component for biodiesel production. The freshly brewed coffee produces more yield compared to commercial RTD coffee might be caused by the difference of roasting condition applied initially on the coffees [6]. The amount of solvent recovered was different due to source difference, $98 \mathrm{ml}$ of hexane was recovered from freshly brewed coffee and $70 \mathrm{ml}$ of hexane was recovered from commercial RTD coffee. The solvent recovery yields achieved were $23 \%$ for commercial RTD coffee and 33\% for freshly brewed coffee as shown in Table 1. The colour extracted from both coffees are different due to diverse sources. 
Table 2. GCMS evaluation result of the coffee oil compositions from coffee sample

\begin{tabular}{|c|c|c|c|}
\hline Peak No. & Retention Time (min) & Compounds & Area \\
\hline \multicolumn{4}{|c|}{ Coffee oil compositions from RTD coffee } \\
\hline 1 & 13.459 & \multirow{7}{*}{ Palmitic Acid } & $0.42 \%$ \\
\hline 2 & 13.9 & & $0.55 \%$ \\
\hline 3 & 14.134 & & $31.49 \%$ \\
\hline 4 & 14.532 & & $2.75 \%$ \\
\hline 5 & 14.625 & & $10.20 \%$ \\
\hline 6 & 14.973 & & $9.74 \%$ \\
\hline 7 & 15.463 & & $1.66 \%$ \\
\hline 8 & 16.011 & Undecanoic Acid & $19.02 \%$ \\
\hline 9 & 16.935 & Linoleic Acid & $0.74 \%$ \\
\hline 10 & 17.383 & Stearic Acid & $16.71 \%$ \\
\hline 11 & 17.525 & Pentadecanoic Acid & $2.72 \%$ \\
\hline 12 & 17.717 & Stearic Acid & $0.85 \%$ \\
\hline 13 & 19.011 & Cinnamyl Cinammate & $0.38 \%$ \\
\hline \multicolumn{4}{|c|}{ Coffee oil compositions from freshly brewed coffee } \\
\hline 1 & 13.487 & Methyl; Palmitate & $3.24 \%$ \\
\hline 2 & 13.915 & Methyl Palmitate & $2.80 \%$ \\
\hline 3 & 14.625 & Methyl Linolelaidate & $3.38 \%$ \\
\hline 4 & 14.767 & Methyl Stearate & $2.67 \%$ \\
\hline 5 & 15.037 & Methyl linoleate & $2.67 \%$ \\
\hline 6 & 16.004 & Undecanedioic Acid & $18.89 \%$ \\
\hline 7 & 16.11 & Adipic Acid & $3.97 \%$ \\
\hline 8 & 17.419 & Butyl 9,12-octadecadienoate & $21.93 \%$ \\
\hline 9 & 17.554 & o-Xylene-d10 & $6.80 \%$ \\
\hline 10 & 17.902 & Benzene & $4.36 \%$ \\
\hline 11 & 18.257 & Naphthalene & $4.92 \%$ \\
\hline 12 & 18.343 & Ferrocene & $4.72 \%$ \\
\hline 13 & 18.527 & 2-(2-Hydroxyphenyl) buta-1,3-diene & $2.95 \%$ \\
\hline 14 & 18.684 & 12-Methoxy-3-methylcholanthrene & $5.12 \%$ \\
\hline 15 & 18.854 & pseudo gem-acetyl(chloromethyl)(2.2)PARACYclophane & $2.84 \%$ \\
\hline 16 & 18.961 & E-1-Diphenyl phosphinoyl-3-methylpent-3-en-2-one & $2.83 \%$ \\
\hline 17 & 19.501 & Linoleic Acid & $2.89 \%$ \\
\hline 18 & 31.586 & Palmitic Acid/ 4-nitro palmitate & $2.92 \%$ \\
\hline
\end{tabular}

In other words, coffee from freshly brewed produces $13 \mathrm{ml}$ of coffee oil and commercial RTD coffee produces $8 \mathrm{ml}$ of coffee oil. After the coffee oil is collected, it will be separated for sample analysis. Highest coffee oil yield achieved was $12 \%$ from freshly brewed coffee samples. Meanwhile, the commercial RTD coffee sample achieved $10 \%$ of the coffee oil yield.

\subsection{Coffee Oil Analysis}

Fatty acid is required to produce biodiesel. The fatty acids include Palmitic acid, Linoleic Acid, and Oleic Acid. In this research the coffee oil samples were analyzed using GCMS analysis to investigate the fatty acid (FFA) content in the coffee oil. The GCMS analysis is divided into 4 parameters as explained in the design of the experiment. The signals detected in GCMS were observed and analysed. For coffee oil analysis the objective was to find traces of fatty acid (FFA) in the samples. Since the fatty acid was the basic roots of fatty acid methyl ester (FAME) or biodiesel, higher FFA content in the coffee oil sample would be a good indication of biodiesel production in this research. The coffee oil obtained from the commercial RTD coffee sample was analyzed. There were 16 components detected in the form of peaks with different retention time according to the composition. The fatty acid in nonsaponified commercial RTD coffee shows that the major fatty acid content was in the form of palmitic acid, contributing in a total of $56.81 \%$.

GCMS has detected fatty acids as the major content of the coffee oil solution (Table 2). In total there were $96.85 \%$ fatty acid content in the sample with the composition of palmitic acid 55.84\%, Undecanoic acid $19.02 \%$, Linoleic Acid $0.74 \%$, Pentadecanoic acid $2.72 \%$, and Octadecanoic acid $0.85 \%$. The quantitative analysis was determined based on the area of GCMS peaks, and is a reflection of the amount of a specific 
analyte present in the sample. In the GC/MS chromatogram, the area will be based on the number of counts taken by the mass spectrometer detector at the point of retention [7].

Therefore, the determination of percentage was according to the area detected in the GCMS spectrum and repeated composition was characterized as one, in this case palmitic acid were added and summed to show the amount of palmitic acid present in the commercial RTD coffee sample. Biodiesel is prone to oxidation due to unsaturated fatty acid [8]. Oxidation occurred in biodiesel with an indication of double bonds present in the molecules. However, the major content in the commercial RTD coffee has shown that Palmitic Acid, and Undecanoic Acid was a single bounded formula, indicating lower oxidation level in biodiesel, while stearic acid was a double bounded formula that indicated the oxidation activity occur in this sample.

The freshly brewed sample indicated a lower amount of fatty acid in comparison to the commercial RTD coffee. The total composition of FFA in the sample was $75.45 \%$. The major fatty acid content for freshly brewed coffee samples were Stearic Acid and Undecanoic Acid which contributed $43.6 \%$ in the total sample composition which can be seen on Table 2 . The difference distribution of FFA from both samples is probably due to the initial composition of each coffee type which are different. Freshly brewed coffee uses coffee beans directly in the brewing process, meanwhile commercial RTD coffee is produced by mixing grinded coffee beans together with other ingredients. In the industrial coffee sachet production, several types of coffee were prepared through a food processing such as spray drying which could affect the quality of the coffee products.

In terms of oxidation activity, the major content was stearic acid which has the double bound structures. Hence, for the freshly brewed coffee samples were considered to have a high oxidation activity in the sample.

\subsection{Direct Transesterification Reaction}

The transesterification process performed parallelly when the coffee oil sample was analysed. Therefore, the composition result of coffee oil was not yet examined during the esterification reaction. Esterification reaction occurs for all samples including freshly brewed coffee and commercial RTD coffee sample.

The parameters performed were according to the literature with the best result according to the previous research [4]. After the esterification process the product cooled down overnight. Later the upper layer which is biodiesel separated with glycerine in the bottom layer.

After the trans-esterification process, both samples were dried up and precipitated in the rounded bottom flask that has been used for esterification reaction. Both samples changed from liquid into solid. The expected result of transesterification was the coffee oil transforms into biodiesel in liquid form. After the esterification the biodiesel and the glycerine were separated by density difference. However, since the liquid product was turned into solid form, it was not considered as biodiesel, thus was not included in the GCMS analysis. The possibilities of high free fatty acid in the sample indicated that direct transesterification was not possible and considered as a failure. The formation of soap might happen if the oil contains $>1 \%$ wt FFA when it undergoes alkalinecatalyzed transesterification reaction [9].

\subsection{Direct Esterification Reaction}

Since the direct transesterification process was not successful, the direct esterification process was performed. The esterification reaction was performed under reflux conditions for 3 hours at $65^{\circ} \mathrm{C}$, with $1: 9$ ratio of coffee oil and methanol-sulfuric acid solution. In this research, $5 \mathrm{ml}$ coffee oil was reacted with $45 \mathrm{ml}$ methanol and $0.6 \mathrm{ml} \mathrm{H}_{2} \mathrm{SO} 4: 0.1 \mathrm{M}$. After the reaction occurred, the esterified product was transferred into a separation funnel to separate the biodiesel and glycerol.

The separation process was done overnight to ensure the upper layer and bottom layer was completely separated. The upper layer which was expected as the biodiesel product was separated with the bottom layer (glycerol) and the biodiesel phase was analysed using GCMS to obtain the biodiesel contents.

Qualitatively, the esterification product for freshly brewed coffee showed dark brown colour of glycerol and concentrated brown oil. Meanwhile, for commercial RTD coffee it produced cream colour of glycerol with light brown oil colour. The biodiesel production achieved for commercial RTD coffee and freshly brewed' coffee were $30 \%$ and $80 \%$.

After the separation of biodiesel and glycerol, the biodiesel was analysed by GCMS for both commercial RTD coffee and freshly brewed coffee.

The results were as expected by the fact that fatty acid methyl ester (FAME) was formed in commercial RTD coffee and freshly brewed coffee samples. The commercial RTD coffee obtained from esterification reaction was analysed in GCMS. The results of the sample analysis in the GCMS spectrum in total were 18 components detected. The FAME formed in this sample was $78.68 \%$, where Methyl Stearate contributes $41.81 \%$ as the major FAME content, followed by Methyl Palmitate with $30.44 \%$. 
Table 3. GCMS evaluation result of the esterified coffee oil compositions from coffee sample

\begin{tabular}{|c|c|c|c|}
\hline Peak No. & Retention Time (min) & Compounds & Area \\
\hline \multicolumn{4}{|c|}{$\begin{array}{l}\text { Esterified coffee oil compositions from RTD coffee } \\
\end{array}$} \\
\hline 1 & 9.688 & Methyl Palmitate & $30.44 \%$ \\
\hline 2 & 10.96 & Methyl Stearate & $34.67 \%$ \\
\hline 3 & 11.097 & Methyl Stearate & $7.14 \%$ \\
\hline 4 & 11.277 & hexadecenoic acid 1-(hydroxymethyl)-1 2-ethanedylester & $1.05 \%$ \\
\hline 5 & 12.106 & Eicosanoic acid methyl ester & $0.80 \%$ \\
\hline 6 & 12.234 & Methyl Eicosanoate methyl ester & $4.30 \%$ \\
\hline 7 & 12.285 & Linoelaidic acid & $1.42 \%$ \\
\hline 8 & 13.858 & pyreno[4,5-c] furan & $1.40 \%$ \\
\hline 9 & 13.286 & Methyl docosanoate & $1.33 \%$ \\
\hline 10 & 13.491 & Benzene & $2.01 \%$ \\
\hline 11 & 13.628 & Methyl 4-ethyl-4'-methoxy-3,5-dimethylbiphenyl-2-carboxylate & $0.61 \%$ \\
\hline 12 & 13.713 & $2,3,4,8$-tetramethyl phenanthrene & $4.01 \%$ \\
\hline 13 & 13.91 & 1-methyltryptophan & $3.91 \%$ \\
\hline 14 & 15.859 & Squalene & $0.66 \%$ \\
\hline 15 & 16.466 & Beta-Tocopherol & $1.09 \%$ \\
\hline 16 & 17.312 & Vitamin E & $0.98 \%$ \\
\hline 17 & 18.911 & Stigmasta-5 & $2.37 \%$ \\
\hline 18 & 19.663 & Gamma-Sitosterol & $1.81 \%$ \\
\hline \multicolumn{4}{|c|}{ Esterified coffee oil compositions from freshly brewed coffee } \\
\hline 1 & 9.986 & Methyl Palmitate & $20.94 \%$ \\
\hline 2 & 11.097 & Methyl Stearate & $23.25 \%$ \\
\hline 3 & 11.225 & Methyl Stearate & $6.30 \%$ \\
\hline 4 & 11.345 & Methyl 10,12 octadecadienoate & $0.26 \%$ \\
\hline 5 & 11.396 & Hexadecanoic Acid & $0.02 \%$ \\
\hline 6 & 11.345 & Glycidyl palmitate & $4.25 \%$ \\
\hline 7 & 12.632 & Methyl Eicosanoate & $3.12 \%$ \\
\hline 8 & 12.414 & Methyl 11-(3-pentyl-2-oxiranyl)undecanoate, cis- & $0.45 \%$ \\
\hline 9 & 13.226 & Butyl 9,12-Octadecadienoate & $5.60 \%$ \\
\hline 10 & 13.354 & cis-9-Hexandecanal & $2.29 \%$ \\
\hline 11 & 13.44 & Methyl Behenate & $1.39 \%$ \\
\hline 12 & 13.499 & Oleoyl Chloride & $1.12 \%$ \\
\hline 13 & 13.576 & 4-Phenyl 3-buten-2-one & $3.72 \%$ \\
\hline 14 & 13.713 & 5,6-dimethyl-Benzimidazole & $12.10 \%$ \\
\hline 15 & 1.3816 & Bicyclo (2.2.1) heptane-acetic acid & $1.86 \%$ \\
\hline 16 & 13.91 & 2,3 dihydro-7,9 methoxy-4 methyl-8-1(methylethyl) & $6.69 \%$ \\
\hline 17 & 14.252 & Combretastatin D-2 methyl ether & $0.88 \%$ \\
\hline 18 & 14.32 & (1-Ethyl Vinyloxy) trimethylsilane & $0.62 \%$ \\
\hline 19 & 14.38 & Ethyl Oleate & $1.00 \%$ \\
\hline 20 & 14.44 & Methyl tetracosanoate & $2.64 \%$ \\
\hline 21 & 15.286 & 3-chlorophenyl hydrazine & $0.69 \%$ \\
\hline 22 & 16.816 & beta-Tocopherol & $0.80 \%$ \\
\hline
\end{tabular}

Based on the fatty acid content that was previously explained (Table 3), through direct esterification reaction the fatty acid conversion into fatty acid methyl ester (FAME) achieved $79 \%$ for the commercial RTD coffee sample. In terms of oxidation activity, natural antioxidants have been detected with the GCMS in the form of Vitamin E and Squalene. It was suspected that traces of natural antioxidant presents were obtained in the factory of the commercial RTD coffee producers.
For esterified freshly brewed coffee there were 22 components detected by GCMS. In comparison to the coffee oil analysis, the freshly brewed coffee samples also have more signal detected than the commercial RTD coffee sample.

FAME was also the major content in freshly brewed coffee samples. In total there were $69.2 \%$ of FAME in freshly brewed coffee samples. In comparison to commercial RTD coffee, freshly brewed coffee produced 
lower amounts of FAME as there were known impurities in freshly brewed coffee samples detected during GCMS analysis which are hexane and ethanol. However, the yield production for freshly brewed showed significant yield difference. For this sample varieties of FAME types were detected, there were 10 different FAME formed in this sample with the major content was Methyl Stearate and Methyl Palmitate contributed $29.55 \%$ and $20.9 \%$.

\section{CONCLUSION}

Spent Coffee Grounds can be utilized for biodiesel production by first drying the SCG and then extracting the oil and later following the common methodology to obtain the biodiesel. Direct transesterification method with alkaline catalyst is not suitable for this procedure as high FFA exists which may produce soap instead of the targeted product. Meanwhile, a direct esterification reaction has been successfully done to convert fatty acid into FAME. The conversion of FFA to FAME in this research achieved $79 \%$ for commercial RTD coffee samples and $91 \%$ for freshly brewed coffee samples

\section{REFERENCES}

[1] E.M. Clarke, E.A. Emerson, Design and synthesis of synchronization skeletons using branching time temporal logic, in: D. Kozen (Eds.), Workshop on Logics of Programs, Lecture Notes in Computer Science, vol. 131, Springer, Berlin, Heidelberg, 1981, pp. 52-71. DOI: https://doi.org/10.1007/BFb0025774

[2] J.P. Queille, J. Sifakis, Specification and verification of concurrent systems in CESAR, in: $M$. Dezani-Ciancaglini and U. Montanari (Eds.), Proceedings of the 5th International Symposium on Programming, Lecture Notes in Computer Science, vol. 137, Springer, Berlin, Heidelberg, 1982, pp. 337-351. DOI: https://doi.org/10.1007/3-54011494-7_22

[3] C. Baier, J-P. Katoen, Principles of Model Checking, MIT Press, 2008.

[4] M. Kwiatkowska, G. Norman, D. Parker, Stochastic model checking, in: M. Bernardo, J. Hillston (Eds.), Proceedings of the Formal Methods for the Design of Computer, Communication and Software Systems: Performance Evaluation (SFM), Springer, Berlin, Heidelberg, 2007, pp. 220-270. DOI: https://doi.org/10.1007/978-3-540-72522-0_6

[5] V. Forejt, M. Kwiatkowska, G. Norman, D. Parker, Automated verification techniques for probabilistic systems, in: M. Bernardo, V. Issarny (Eds.), Proceedings of the Formal Methods for Eternal Networked Software Systems (SFM), Springer,
Berlin, Heidelberg, 2011, pp. 53-113. DOI: https://doi.org/10.1007/978-3-642-21455-4_3

[6] G.D. Penna, B. Intrigila, I. Melatti, E. Tronci, M.V. Zilli, Bounded probabilistic model checking with the muralpha verifier, in: A.J. Hu, A.K. Martin (Eds.), Proceedings of the Formal Methods in Computer-Aided Design, Springer, Berlin, Heidelberg, 2004, pp. 214-229. DOI: https://doi.org/10.1007/978-3-540-30494-4_16

[7] E. Clarke, O. Grumberg, S. Jha, et al., Counterexample-guided abstraction refinement, in: E.A. Emerson, A.P. Sistla (Eds.), Computer Aided Verification, Springer, Berlin, Heidelberg, 2000, pp. 154-169.

DOI: https://doi.org/10.1007/10722167_15

[8] H. Barringer, R. Kuiper, A. Pnueli, Now you may compose temporal logic specifications, in: Proceedings of the Sixteenth Annual ACM Symposium on the Theory of Computing (STOC), ACM, 1984, pp. 51-63. DOI: https://doi.org/10.1145/800057.808665

[9] A. Pnueli, In transition from global to modular temporal reasoning about programs, in: K.R. Apt (Ed.), Logics and Models of Concurrent Systems, Springer, Berlin, Heidelberg, 1984, pp. 123-144. DOI: https://doi.org/10.1007/978-3-642-82453-1_5

[10] B. Meyer, Applying "Design by Contract", Computer 25(10) (1992) 40-51. DOI: https://doi.org/10.1109/2.161279

[11] S. Bensalem, M. Bogza, A. Legay, T.H. Nguyen, J. Sifakis, R. Yan, Incremental component-based construction and verification using invariants, in: Proceedings of the Conference on Formal Methods in Computer Aided Design (FMCAD), IEEE Press, Piscataway, NJ, 2010, pp. 257-256.

[12] H. Barringer, C.S. Pasareanu, D. Giannakopolou, Proof rules for automated compositional verification through learning, in Proc. of the 2nd International Workshop on Specification and Verification of Component Based Systems, 2003.

[13] M.G. Bobaru, C.S. Pasareanu, D. Giannakopoulou, Automated assume-guarantee reasoning by abstraction refinement, in: A. Gupta, S. Malik (Eds.), Proceedings of the Computer Aided Verification, Springer, Berlin, Heidelberg, 2008, pp. 135-148. DOI: https://doi.org/10.1007/978-3-54070545-1_14 